\title{
Response selection and visual search
}

\author{
MURRAY J. WHITE \\ Victoria University of Wellington, Wellington, New Zealand
}

\begin{abstract}
Subjects made forced-choice or go/no-go responses to target letters flanked by noiseincompatible or noise-compatible letters. Noise compatibility had a pronounced effect on RTs, but there was no evidence for any selective effect of type of response on target detection times.
\end{abstract}

Evidence for the view that noise letters impair visual processing at the response selection stage has been advanced by Eriksen and Eriksen $(1974,1979)$ and C. W. Eriksen and Schultz (1979). With two targets $(\mathrm{H}$ and $\mathrm{K})$ assigned to one lever-movement response and two others (C and S) to another lever-movement response, the targets in noise-compatible displays (KKKHKKK) are detected more rapidly than are targets in noise-incompatible displays (SSSHSSS). Response competition effects are extremely powerful and appear to account for a substantially greater amount of variance than effects due to target-noise featural relationship. Even when target and noise stimuli are made compatible with a single response movement, targets flanked by repetitions of the same letter (KKKKKKK) are detected more rapidly than are targets flanked by repetitions of the other letter (CCCKCCC) (Eriksen \& Eriksen, 1979). This finding is not easily accommodated by inhibitory interactive channels models (e.g., Bjork \& Murray, 1977). In all relevant experiments reported to date, subjects have been required to make a forced-choice response, pushing one button or lever whenever a target is a member of one specified set and pushing another button or lever whenever the target is a member of a different set. It is conceivable that this procedure also engenders an element of response competition, in that subjects have to retain an association between two sets of letters and two response movements and make a decision about which of the response movements should be initiated. The possibility that overt response selection somehow interacts with an earlier, internal recognition response selection cannot be discounted (Corballis, Roldan, \& Zbrodoff, 1974). If response selection and internal response competition effects are eliminated or minimized, will effects attributable to interactive inhibition be more pronounced in the Eriksen visual search experiment?

\section{METHOD}

\section{Stimuli}

Target and noise letters were the capitals $\mathrm{C}, \mathrm{K}, \mathrm{H}$, and $\mathrm{S}$. In five sets of 16 displays, each letter appeared four times in the

This research was supported by grants from Victoria University of Wellington $(85 / 80)$ and the University Grants Committee (70/51). center of a stimulus card and the sets were constructed as follows: (1) In no-noise displays, only the centered letter appeared in a card. (2) In noise-compatible and featurally same displays, each centered letter was flanked by three repetitions of the same letter (e.g., KKKKKKK). (3) In noise-compatible and featurally dissimilar displays, each letter was flanked by three repetitions of the other letter from the same response set (e.g., CCCKCCC). (4) In noise-incompatible and featurally similar displays, each letter was surrounded by three repetitions of another letter from the other response set (e.g., HНHKHHH). (5) In noiseincompatible and featurally dissimilar displays, each letter was flanked by three repetitions of another letter from the other response set (e.g., SSSKSSS). In each of four blocks of 20 display cards, there was one instance of each of the four centered letter by five display type arrangements. In multiple-letter displays, the spacing between the edges of adjacent letters was $.26 \mathrm{deg}$, with a display subtending an overall visual angle of $3.38 \mathrm{deg}$.

\section{Procedure}

Stimulus cards within each set were randomized for presentation in a Gerbrands three-field tachistoscope. A constant background illumination of $4.7 \mathrm{~cd} / \mathrm{m}^{2}$ was used throughout. In each trial, two short thin lines, one appearing directly above and one below the position at which the target letter would appear, were shown for $2 \mathrm{sec}$. Immediately on the offset of these fixation marks, a stimulus array was shown for $1 \mathrm{sec}$, and on the offset of this, a blank illuminated field was shown for 5 sec.

Twelve females and 8 males were randomly assigned, 10 to a go/no-go (GNG) and 10 to a two-alternative forced-choice (2AFC) condition. In the former, a response was to be made only if the cued target letter was a member of a specified set, namely, $\mathrm{C}$ and $\mathrm{K}$ for five subjects and $\mathrm{H}$ and $\mathrm{S}$ for the other five subjects. In the $2 \mathrm{AFC}$ condition, one response was to be made if the cued letter belonged to the target set (e.g., $\mathrm{C}$ and $\mathrm{K}$ ) and a different response was to be made if it belonged to the nontarget set (e.g., $\mathrm{H}$ and $\mathrm{S}$ ). In both conditions, a response was made by the subject's depressing two microswitches, one with the left index finger and one simultaneously with the right index finger. The $2 \mathrm{AFC}$ subjects also made left middle and right middle finger responses when the cued letter was a member of the nontarget set. Only index finger responses were monitored in both conditions. The first session of 80 trials and the first 20 trials in the second and third sessions were treated as practice.

\section{RESULTS AND DISCUSSION}

An analysis of the mean correct RTs (Table 1) collapsed over the featural similarity variable showed a strong effect due to type of display $[\mathrm{F}(2,36)=17.65$, $\mathrm{p}<.001]$. Targets in no-noise displays were detected as rapidly as targets in noise-compatible displays $(565 \mathrm{msec}$ and $581 \mathrm{msec}$ ), and these times were significantly faster than those for noise-incompatible displays $(626 \mathrm{msec}$, 
Table 1

Mean Correct Reaction Times (in Milliseconds)

\begin{tabular}{cccccc}
\hline & \multicolumn{4}{c}{ Target-Noise Combination } \\
\cline { 2 - 6 } Response & Noise-Incompatible & & Noise-Compatible & No \\
\cline { 2 - 5 } Condition & S & D & S & D & Noise \\
\hline GNG & 519 & 513 & 473 & 488 & 471 \\
2AFC & 743 & 727 & 687 & 676 & 659 \\
\hline
\end{tabular}

Note $-S=$ similar D = different .

Newman-Keuls test). In both GNG and 2AFC conditions, target redundancy neither facilitated nor impaired target detection (473 vs. $471 \mathrm{msec}$ and 687 vs. $659 \mathrm{msec}$, respectively). Collectively, these results are consistent with an independent-channels conceptualization of visual search (Eriksen \& Eriksen, 1979; Krueger \& Shapiro, 1980). A second analysis of the results for only the noise-compatible and noise-incompatible displays with featural relationship included as a factor showed no effect whatsoever due to target-noise featural similarity $(F<1)$. Contrary to what might have been expected, RTs were marginally slower for featurally different noise-compatible displays in the GNG condition. Overall, RTs in the simple response condition were much faster than those in the forced-choice condition $[F(1,18)=16.95, p<.001]$, but type of response nowhere interacted with any other variable. Mean error rates were $.5 \%$ in the GNG condition and $3.3 \%$ in the $2 \mathrm{AFC}$ condition.

The absence of a featural relationship effect is equivocal, in that Eriksen and Eriksen (1979) have reported faster RTs for noise-same-as-target displays but the postulates of inhibitory interactive models necessarily predict the opposite, that is, faster RTs for noise/ alternative-target displays. Speculatively, this null finding might represent the interdependent effects of featural relationship and response competition. Any benefits conferred on targets in CCCKCCC displays at an early feature extraction stage are cancelled and vitiated by competition among internal recognition responses at a later response decision stage. This explanation is post hoc, and while it is impossible to identify separate processing times for each of these stages, it is of some interest to note that in the GNG condition 8 of the 10 subjects made faster RTs for noise-same-as-target displays and that these RTs approximated the values reported by Eriksen and Eriksen (1979, Experiment 1). Overall, RTs were slowest in the $2 \mathrm{AFC}$ noise-incompatible condition, and here, targets in featurally different displays were detected faster than targets in featurally similar displays (see Table 1). In short, however, the study has provided no evidence for any selective effect of response procedure on target detection in the Eriksen visual search design.

\section{REFERENCES}

BJork, E. L., \& Murray, J. T. On the nature of input channels in visual processing. Psychological Review, 1977, 84, 472-484.

Corballis, M. C., Roldan, C. E., \& Zbrodoff, J. Response set effects in recognition memory. Memory \& Cognition, 1974, 2, 501-508.

Eriksen, B. A., \& Eriksen, C. W. Effects of noise letters upon the identification of a target letter in a nonsearch task. Perception \& Psychophysics, 1974, 16, 143-149.

Eriksen, C. W., \& Eriksen, B. A. Target redundancy in visual search: Do repetitions of the target within the display impair processing? Perception \& Psychophysics, 1979, 26, 195-205.

Eriksen, C. W., \& Schultz, D. W. Information processing in visual search: A continuous flow conception and experimental results. Perception \& Psychophysics, 1979, 25, 249-263.

Krueger, L. E, \& Shapiro, R. G. Repeating the target neither speeds nor slows its detection: Evidence for independent channels in letter processing. Perception \& Psychophysics, 1980, 28, 68-76.

(Received for publication July 16, 1981.) 